\title{
Mathematical Study of Medicine Propagation in Biological Tissue and Some of Its Applications
}

\author{
N. G. Gulko1, I. T. Selezov², R. I. Volinsky³ \\ ${ }^{1}$ Department of Mathematics, Ben-Gurion University of the Negev, Beer-Sheva, Israel \\ ${ }^{2}$ Institute of Hydromechanics, NAS of Ukraine, Kiev, Ukraine \\ ${ }^{3}$ Department of Geography, Ben-Gurion University of the Negev, Beer-Sheva, Israel \\ Email: nataliya.gulko@gmail.com
}

How to cite this paper: Gulko, N.G., Selezov, I.T. and Volinsky, R.I. (2021) Mathematical Study of Medicine Propagation in Biological Tissue and Some of Its Applications. Journal of Applied Mathematics and Physics, 9, 127-132.

https://doi.org/10.4236/jamp.2021.91009

Received: November 27, 2020

Accepted: January 23, 2021

Published: January 26, 2021

Copyright (c) 2021 by author(s) and Scientific Research Publishing Inc. This work is licensed under the Creative Commons Attribution International License (CC BY 4.0).

http://creativecommons.org/licenses/by/4.0/

\begin{abstract}
The paper deals with the problem of the distribution of the medicine (enzyme) in the damaged biological tissue where the reaction enzyme-substrat takes place. The biological problem is reduced to a singular degenerate initial-boundary value problem for two coupled ordinary differential equations. Analytical solution of the singular degenerated IBV-problem was obtained by power series. The solution demonstrates the real situation and found suitable to depict the degeneration of singular system, caused by low concentration of the enzyme.
\end{abstract}

\section{Keywords}

Enzyme, Substrate, Reaction, Analytical Solution, Numerical Solution, Singular Degeneration, Initial Boundary Value Problem

\section{Introduction and Auxiliary Results}

There are many different works dealing with medicine (enzyme) transportation to the damaged zone. The mathematical modeling of biological processes often leads to highly complex systems involving many state-variables and reactions. For a wide range of analysis model complexity can present an insurmountable barrier [1]. Methods of model reduction therefore remain a vital topic and a widely applicable tool in the analysis and modeling of biochemical system. To describe a biochemical reaction of medicine, injected into the tissue, we present here mathematical model [2]. The model fully reflects the formation of the enzyme-substrate complex in the damaged tissue [3] [4]. It makes possible to demonstrate the real situation and found suitable to depict the degeneration of singular system, caused by low concentration of the enzyme. The same mathemati- 
cal model was considered also in the previous work [5]. The analytical solution of the corresponding Cauchy problem using the power series method and numerical solutions using the Runge-Kutta-Felberg method [6] were obtained for a large time scales. Some other authors [7] discus changes in substrate concentration (decreases with time) and enzyme-extract complex (increases with time). On an example the natural rubber bio synthesis process using the Michaelis-Menten model the quasi stationary states with constant rates of intermediate reactions in a chain were obtained. Conditions of convergence are developed for different situations in the case of a biochemical reaction were examined [8]. In other work "Extensions to Michaelis-Menten Kinetics for Single Parameters" [9] they defined the equation for substrate and product and rate of reaction based on rate and time perspectives. Two quadratic equations were developed. The first, represents a body entity that gave a useful relationship of enzyme and the second of nutrients. By combining Enzymes and Enzyme-substrate complexes values, they derive a Michaelis-Menten hyperbolic function and the proportionate rate of reaction and Enzymes values of the quadratics resulting in another Michaelis-Menten hyperbolic. It was obtained that between these two hyperbolic functions, in-competitive inhibitions exist, indicating metabolic activities and growth in terms of energy levels. The effect of decomposition to the kinetics of the enzyme reaction based on a microscopic pharmacokinetic model and the application of the Monte Carlo method to analyze that explains possible reaction scenarios in disordered media using the kinetics of the Michaelis-Menten model in pharmacology [10]. In [11] complex variables method combined with Monte Carlo method.

It this paper we provide the overview of the main methods proposed for an analytical solution. The solution of the Cauchy problem with initial conditions is given for two functions $x(t)$ and $y(t)$, which is characterized by a singular degeneracy with respect to the parameter $\epsilon$ (Section 2). An analytical method for representing the solution by power series was proposed. The functions in power series up to the fifth order were calculated (in Section 3). On this basis, solutions for changes in the concentration of the substrate and enzyme-substrate complex were obtained (in Section 4). Accordingly, when using the Runge-Kutta-Felberg method, calculations were carried out up to the $9^{\text {th }}$ order. In the above analysis, in contrast to previous studies, it is not required to take into account the limitations on the very small amount of the introduced enzyme $\epsilon$.

\section{The Problem Definition}

The mathematical model can be represented as the Cauchy problem for a system of coupled ordinary differential equations for two functions $x(t), y(t)$

$$
\begin{gathered}
\frac{\mathrm{d} x}{\mathrm{~d} t}=-x+(x+\mu-\lambda) y \\
\epsilon \frac{\mathrm{d} y}{\mathrm{~d} t}=x-(x+\mu) y
\end{gathered}
$$

with the following initial conditions 


$$
x(0)=1, y(0)=0
$$

Equations (2.1) and (2.2) are given in dimensionless form according to the formulas

$$
t^{*}=k_{0} e_{0} t, \quad \lambda=\frac{k_{2}}{k_{1} s_{0}}, \quad \mu=\frac{k_{-1}+k_{2}}{k_{1} s_{0}}, x(t)=\frac{s(t)}{s_{0}}, y(t)=\frac{c(t)}{e_{0}}, \quad \epsilon=\frac{e_{0}}{s_{0}}
$$

There $t$ it time, $s, e$ and $c$ are the concentrations of the substrate, enzyme and substrate-enzyme complex, $s_{0}, e_{0}, c_{0}$ are relevant initial concentrations, $k_{1}$ and $k_{-1}$ describe the rates of the forward and the reverse reactions, $k_{1}$ is rate of the second reaction. The asterisks are omitted everywhere in formulas for dimensionless variables.

\section{Analytical Solution for Arbitrary Functions}

Equations (2.1) and (2.2) are strongly coupled with nonlinear terms. So, to construct an analytical solutions for the Cauchy problem is not trivial. In this work, we use the Michaelis-Menten method for a general case with no restrictions on the initial enzyme concentration. For solution of the corresponding Cauchy problem we use a power series method, applying to the time coordinate. The method is valid for a short interactions, wihout any restrictions on the enzime concentration. Now, more relative terms were held in the expansion, than in the in series in earlier works [5]. The second approach is the numerical solution of the problem by the Runge-Kutta-Felberg method, which is valid for long time intervals and large values of parameters and concentrations, i.e., for strong interactions when the values of the enzyme and enzyme-substrate complex are of the same order. We present the results in dimensionless form. The results are suitable for interactions in the case of any real enzyme and substrate structures. Usually, the medicine transported for a sufficiently large distance, the concentration of the medicine is significantly decreases. Consequently, the parameter is characterized by the initial concentration of the enzyme for which the initial reaction is very weak. In this case, problem (2.1) and (2.3) is singularly degenerate, since in this case the coefficient of derivative is very small, and the concentration of the enzyme is low, and an approximate solution to the singularly degenerate problem can be obtained by the method of coalescence asymptotic expansions [4]. In the case when the medicine (enzyme) is distributed in the immediate Near the point where the concentration is large and no singular degeneration appears, we consider the problem (2.1) and (2.3) for arbitrary parameters. Then the problem contains strongly nonlinear systems of differential Equations (2.1) and (2.2) and becomes significantly more complicated.

In this case, the concentrations of the substrate and enzyme are of the same order $s_{0} \cong e_{0}$. The order $\epsilon=O(1)$ and initial reaction rate can be calculated using the corrected formula:

$$
V_{0} \cong \frac{k_{2}}{k_{-1}+k_{2}} k_{1} e_{0} s_{0}
$$

Below we present solutions as a series. without restrictions on the concentra- 
tion of enzyme. With the initial conditions (2.3), we obtain the following series

$$
x(t)=\sum_{k=0}^{k=\infty} a_{k} t^{k}, y(t)=\sum_{k=0}^{k=\infty} b_{k} t^{k}
$$

For the small $t$ values those series will converge. From the initial conditions (2.3) it follows that $a_{0}=1, b_{0}=0$ and, and then series (3.4) can be represented in the form

$$
\begin{aligned}
& x(t)=\sum_{k=0}^{k=\infty} a_{k} t^{k} \\
& y(t)=\sum_{k=1}^{k=\infty} b_{k} t^{k}
\end{aligned}
$$

As we can see, representations (3.5) and (3.6) already satisfy the initial conditions ((3.4). After substituting expansions (3.5), (3.6) into equations (2.1), (2.2), we obtain a system of equations containing terms with increasing powers of $t$. By comparing the coefficients for the same powers of $t$, we obtain expressions for the coefficients $a_{k}$ and $b_{k}$. Keeping three terms in expansions (3.5) and (3.6) we have

$$
\begin{gathered}
a_{1}=-1, a_{2}=\frac{1}{2}-t+\frac{1}{2}(1+\mu-\lambda) \frac{1}{\epsilon} \\
a_{2}=-\frac{1}{2} \frac{1}{\epsilon}-\frac{1}{2}(1+\mu) \frac{1}{\epsilon}
\end{gathered}
$$

As a result, for the functions $x(t)$ and $y(t)$ we obtain:

$$
x(t)=1-t+\frac{1}{2}\left[1+\frac{1}{\epsilon}(1+\mu-\lambda)\right] t^{2}
$$

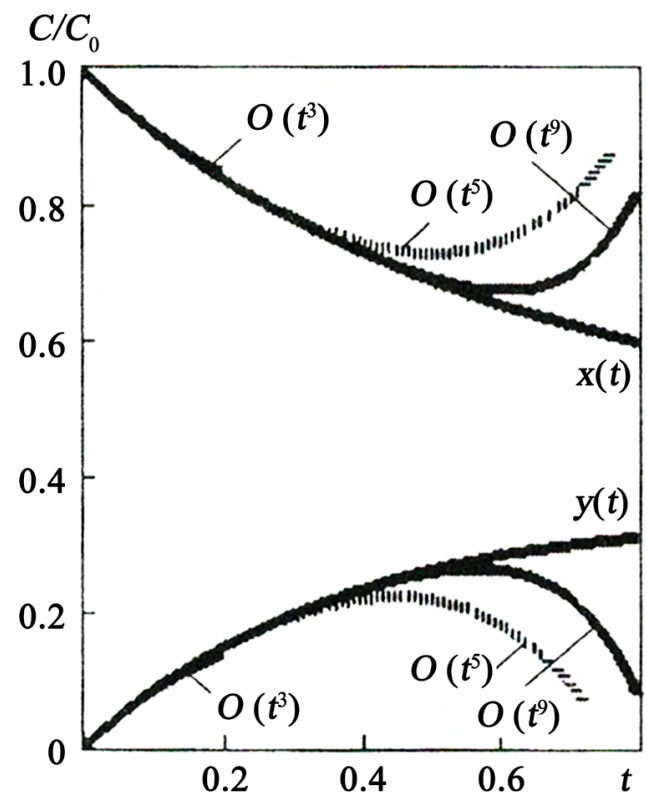

Figure 1. Comparison of the results obtained by the numerical Runge-Kutta Felberg method and the power series approach. 


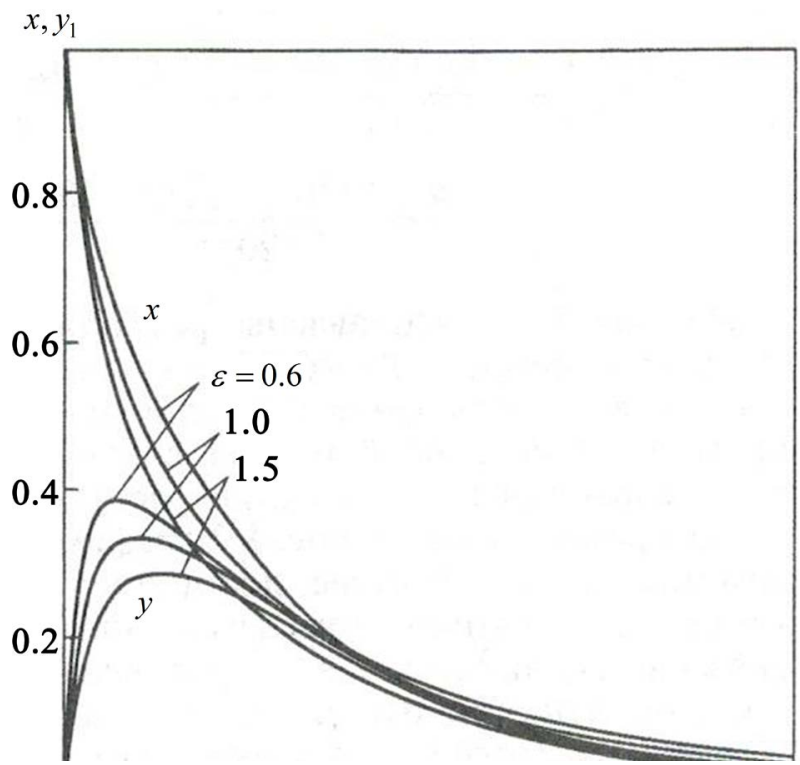

Figure 2. The change in the concentration of the substrate $x(t)$ and the enzyme-substrate comlex $y(t)$ with $\mu=1.0$ and $\lambda=0.5$.

$$
y(t)=\frac{1}{\epsilon} t-\frac{1}{2 \epsilon}\left[1+\frac{1}{\epsilon}(1+\mu)\right] t^{2}
$$

According to (3.4), (3.5) we preserve more terms than (3.7) and (3.8) $(k=$ 3.5... 9). Expressions are also obtained for the coefficients $a_{3}, a_{4}, a_{5}$ and $b_{3}, b_{4}, b_{5}$ (not presented in the current paper)

\section{Analysis of Results}

In Figure 1, the results of the numerical Runge-Kutta-Felberg method and the power series approach are presented and compared. According to the results, we conclude that for relatively short time periods, the series method gives very good approximation, and may be used for descriptions of the expected medicine distribution, characterize the features of its transportation, subsequent localization and its possible impact. From Figure 2, it can be seen that for smaller $\epsilon$ value provides maximum concentration of the enzyme substrate complex. The maximum values increase with decreasing of $\epsilon$.

\section{Conflicts of Interest}

The authors declare no conflicts of interest regarding the publication of this paper.

\section{References}

[1] Snowden, T.J., van der Graaf, P.H. and Tindall, M.J. (2017) Methods of Model Reduction for Large-Scale Biological Systems: A Survey of Current Methods and Trends. Bulletin of Mathematical Biology, 79, 1449-1486. https://doi.org/10.1007/s11538-017-0277-2

[2] Selezov, I.T., Volynski, R.I. and Gulko, N.G. (2009) Modeling Wave Propagation in 
Complex Media. In: Selezov, I.T., Volynski, R.I. and Gulko, N.G., Eds., Modeling Wave Propagation in Complex Media, 3-78.

[3] Michaelis, N. and Menten, M.I. (1913) Die Kinetik der Invertingwirkung. Biochemistry Zeitung, 49, 333-369.

[4] Murray, J.D. (1977) Lectures on Nonlinear-Differential-Equations Models in Biology. Clarendon Press, Oxford.

[5] Selezov, I.T. (2009) Mathematical Modeling and Physiological Aspects. In: Selezov, I.T. and Bersenev, V.A., Eds., Neurometamerism. Mathematical Modeling and Physiological Aspects (In Russian), 3-78.

[6] Selezov, I., Bersenev, V. and Morozova, L. (2011) Modeling of Enzyme-Substrate Reaction in Tissue (In Russian). Sci. Collection "Physics-Mathematical Modeling and Informational Technologies, 13, 132-138.

[7] Vasin, A.A., Gaponenko, A.K., Teplov, A.E. and Markeev, A.N. (2006) Mathematical Modeling Biochemical Reactions on Example of Biosinthesis of Caoutchouc (In Russian). Mathematical Biology and Bioinformatics, 1, 41-57.

https://doi.org/10.17537/2006.1.41

[8] Kosmidis, K., Karalis, V., Argyraki, P. and Macheras, P. (2004) Michaelis-Menten Kinetics under Spatially Constrained Conditions: Application to Mibefradil Pharmacokinetics. Biophysical Journal, 87, 1498-1506. https://doi.org/10.1529/biophysj.104.042143

[9] Ariyawansha, R.T.K., Basnayake, B.F.A., Karunarathna, A.K. and Mowjood, M.I.M. (2018) Extensions to Michaelis-Menten Kinetics for Single Parameters. Scientific Reports, 8, Article Number: 16586. https://doi.org/10.1038/s41598-018-34675-2

[10] Selezov, I.T., Kryvonos, Yu.G. and Gandzha, I.S. (2018) Wave Propagation and Diffraction. Mathematical Methods and Applications. Springer. In Series Foundations of Engineering Mechanics, 237. https://doi.org/10.1007/978-981-10-4923-1

[11] Stay, M.S., Xu, J., Randolph, T.W. and Barocas, V.H. (2003) Computer Simulation of Convective and Diffusive Transport of Controlled-Release Drugs in the Vitreous Tumor. Pharmaceutical Research, 20, 96-102. https://doi.org/10.1023/A:1022207026982 